\title{
Réponses à la stimulation lumineuse modulée sinusoïdalement chez l'homme normal et pathologique.
}

\author{
D. N. J. Donker. \\ Département d'Électroneurologie (Dir. W. STORM VAN LeEuWEN) \\ Université d'Utrecht Nic. Beetsstraat 24 Utrecht, Pays-Bas.
}

\section{INTRODUCTION.}

En pratique courante, on emploie la stimulation avec des éclairs de lumière périodiques pour obtenir des réponses dans le système visuel chez l'homme. Cette méthode a deux désavantages : $1^{\circ}$ L'intensité de lumière moyenne est en rapport avec la fréquence des éclairs et $2^{\circ}$ des éclairs périodiques peuvent être composés d'un grand nombre d'ondes sinusoïdales harmoniques (théorème de Fourier). Aussi les réponses à ces éclairs peuvent contenir des composantes harmoniques, qui peuvent être une conséquence de la présence des composantes harmoniques dans le stimulus de sorte que ces composantes ne sont pas forcément d'origine visuelle.

C'est pourquoi au cours d'une recherche concernant la linéarité et la non-linéarité du système visuel chez l'homme, VAN der Tweel et VerduYN Lunel (1965) ont utilisé une forme de stimulation où l'intensité de la lumière est modulée sous la forme d'un sinus autour d'une intensité de lumière constante (L.M.S.). En faisant augmenter les amplitudes de la modulation sinusoïdale, la profondeur de modulation augmente, sans qu'interviennent de changements dans l'intensité de lumière moyenne. De cette façon, avec plusieurs profondeurs de modulation, des réponses occipitales ont été obtenues, qui montraient, surtout entre 9 et $15 \mathrm{~Hz}$, une grande analogie avec le sinus de stimulation. Une analyse quantitative des réponses enregistrées sur le crâne était ainsi possible, comme SPEKREYSE (1966) et REGAN (1968) l'avaient montré.

A cause de ces propriétés cette forme de stimulation lumineuse semblait offrir des avantages pour 1'application des P.E.V. au diagnostic clinique.

KAMPHUISEN (1969) fut un des premiers à appliquer la méthode au cours d'une recherche sur la signification clinique des réponses cérébrales asymétriques recueillies sur des aires homologues.

Il distingue deux formes d'asymétrie :

a) une asymétrie « réelle » consistante, qui serait fondée sur des perturbations cérébrales unilatérales de nature fonctionnelles et

b) une asymétrie « inconsistante », cette dernière représentant pour l'auteur la conséquence d'un phénomène qu'il a appelé « diminution paradoxale ». Sous ce terme KAMPHUISEN entend la diminution des amplitudes des réponses qui accompagnent une augmentation de la profondeur de modulation. Comparant un groupe de sujets normaux et un groupe de malades, des « asymétries inconsistantes » ont été trouvées chez 16 p. 100 des normaux examinés et chez 54 p. 100 des malades. Dans le groupe des malades une corrélation a été trouvée entre la présence de ces asymétries inconsistantes et l'existence de phénomènes E.E.G. irritatifs.

Tirés à part : D. N. J. Donker (à l'adresse ci-dessus). 
La recherche de KAMPHUISEN conduisait aux hypothèses suivantes :

1. L'apparition d'asymétries « inconsistantes » chez un pourcentage non négligeable de sujets normaux suggère que de telles asymétries - et par conséquent le phénomène de diminution paradoxale - pourraient être causées par la variabilité des amplitudes des réponses.

2. Si les variabilités jouaient un rôle, on pourrait s'attendre à trouver une différence dans la variabilité des amplitudes chez les patients avec anomalies E.E.G. irritatives et chez les sujets normaux.

3. Le degré de variabilité des réponses pourrait donc être un paramètre utilisable dans le diaggnostic clinique.

Dans la recherche rapportée ici, nous avons voulu étudier la reproductibilité des réponses à la L.M.S. et de leurs composantes harmoniques chez des individus normaux et chez des patients afin de déterminer si la méthode peut être utilisée pour l'application au diagnostic clinique.

\section{Méthode.}

Cette étude porte d'une part sur cinq sujets indemnes de tout trouble visuel ou neuro-psychiatrique et d'autre part sur quatre patients chez qui l'E.E.G. de routine montrait des anomalies irritatives c'est-à-dire des pointes, des pointes-ondes et des activités paroxystiques aiguës.

Les électrodes appliquées sur le crâne selon le système 10-20 international, avaient $\mathrm{C}_{\mathrm{o}}$ comme référence commune. Les neuf dérivations utilisées pour l'E.E.G. sont données dans la figure 1 , une $10^{\circ}$ et une $11^{\circ}$ dérivation étaient respectivement enregistrées, le stimulus lumineux et le « trigger » du sinus.

Quant au choix de $C_{0}$ comme électrode de référence, on peut remarquer quə cette localisation a l'a vantage de n'être pas trop influencée par l'électrorétinogramme, l'électro-oculogramme et l'électromyogramme de la musculature frontale. Cependant un inconvénient est qu'en $\boldsymbol{C}_{0}$ des réponses aux stimuli lumineux sont parfois enregistrées. Les dérivations $F_{p} 2-C_{0}$ et $F_{p} 2-A_{2}$ ont été enregistrées comme contrôle (cf. fig. 1) : dans la mesure où aucune réponse n'était enregistrée sur ces dérivations on pourrait conclure à l'absence de réponse puisqu'il n'est pas probable que les électrodes $F_{p} 2, A_{2}$ et $C_{0}$ soient équipotentielles et dans ce cas les enregistrements ainsi obtenus étaient utilisés pour l'analyse.

Les données ont été enregistrées sur un appareil E.E.G. de 16 canaux (type Elema) ayant une bande passant de $0,3-700 \mathrm{~Hz}$ et simultanément sur bande magnétique (type Ampex) ; bande de fréquence 0-500 Hz. La stimulation a été faite à l'aide d'une lampe d'une surface de $60 \times 60 \mathrm{~cm}$, laquelle donnait une lumière diffuse - posée à une distance de $25 \mathrm{~cm}$ du sujet.

L'intensité Iumineuse auprès de l'œil était de $\pm 90 \mathrm{~J}$ lux. Le sujet était stimulé dans la position couchée.

Pour obtenir les réponses les moins compliquées possibles, les fréquences dé stimulation utilisées étaient de 10,12 et $16 \mathrm{~Hz}$. Pour étudier le phénomène de «diminution paradoxale " les profondeurs de la modulation étaient de 10,30 et 50 p. 100 .

Aussi bjen chez les sujets de contrôle que chez les patients, on a toujours effectué le même type d'expérience et ceci quatre fois de suite avec une courte pause entre chaque série. Au cours de chaque expérience, la réponse moyenne a été obtenue avec 100 périodes de stimulations. Le traitement numérique des signaux a consisté en une analyse de Fourier discràte, comme celle décrite par Lopes DA Silva (1970).

Pour chaque sujet à partir des 4 expériences identiques, la moyenne et l'écart type ont été calculés, aussi bien pour l'amplitude globale, que pour l'amplitude des composantes harmoniques. En fait le coefficient de variation a été utilisé, l'écart-type ayant été calculé en pourcentage en ramenant sa valeur à celle de la moyenne.

Le même calcul statistique a été effectué pour chaque paramètre du stimulus et pour chaque région - occipitale, pariétale et temporale droite et gauche - ainsi que pour le groupe total des patients et des sujets normaux (variabilité inter-individuelle).

\section{Sujets normaux.}

\section{RÉSULTATS.}

Distribution topographique. Chez tous les sujets et dans toutes les régions explorées on a observé des réponses, qui présentaient une forme sinusoïdale mais qui ont aussi des composantes harmoniques.

Les régions occipitale et pariétale présentent des réponses de forme différente de celles de la région temporale. La figure 2 montre un exemple de la forme des réponses et de leur distribution topographique chez un sujet normal, obtenues avec des stimulations de $10 \mathrm{~Hz} 30 \mathrm{p} .100$. 


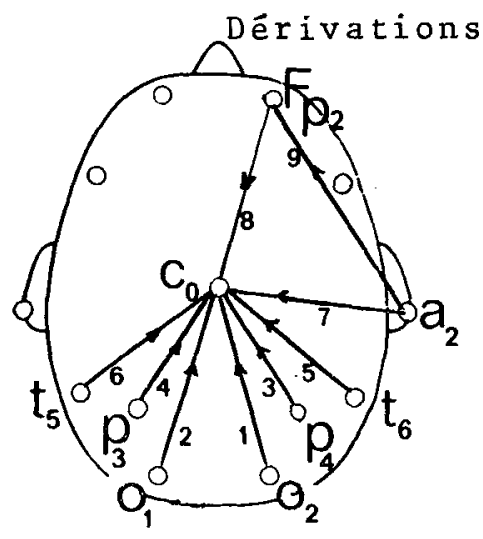

$1: 0_{2} \rightarrow \mathrm{C}_{\mathrm{O}}$

2: $0_{1} \rightarrow \mathrm{C}_{0}$

3: $\mathrm{P}_{4} \rightarrow \mathrm{C}_{0}$

4: $\mathrm{P}_{3} \rightarrow \mathrm{C}_{0}$

5: $\mathrm{T}_{6} \rightarrow \mathrm{C}_{0}$

6: $\mathrm{T}_{5} \rightarrow \mathrm{C}_{\mathrm{o}}$

7: $\mathrm{A}_{2} \rightarrow \mathrm{C}_{\mathrm{o}}$

8: $\mathrm{FP}_{2} \rightarrow \mathrm{C}_{\mathrm{o}}$

$9: \mathrm{Fp}_{2} \rightarrow \mathrm{A}_{2}$

10: Triggersinus

11: Signal de phototube

Fig. 1. - Localisation et montage des électrodes.
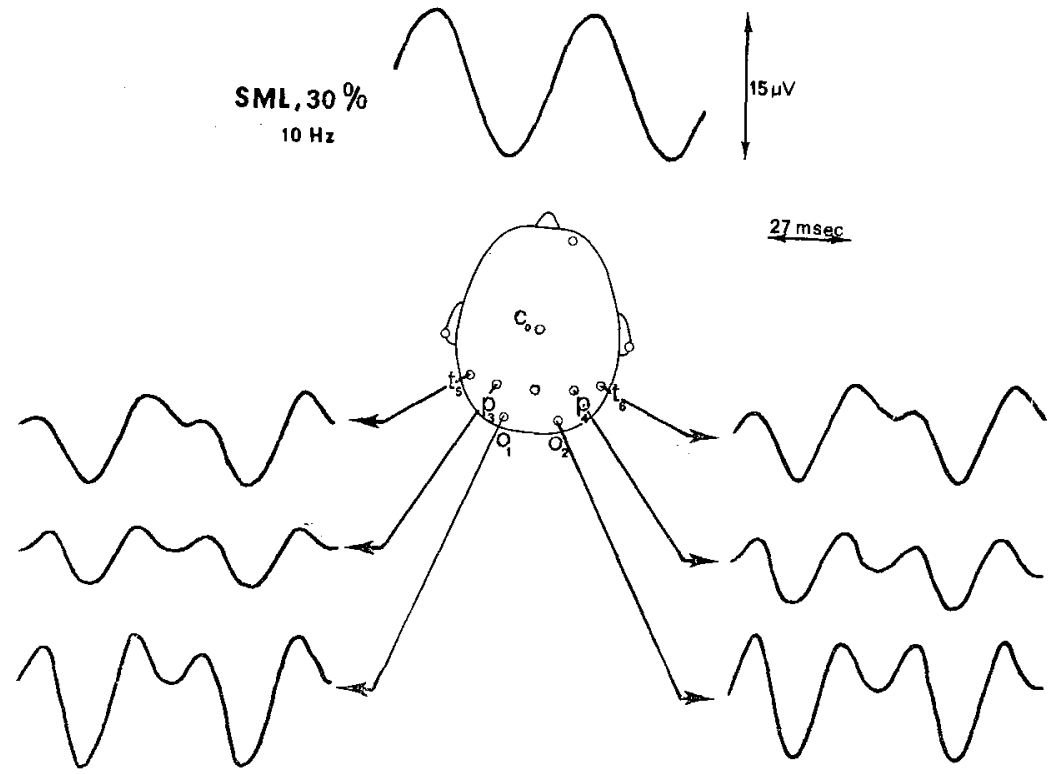

Fig. 2. - Distribution topographique des réponses à la L.M.S. Sujet normal. 


\section{SOCIÉTÉ D'E.E.G. ET DE NEUROPHYSIOLOGIE CLINIQUE DE LANGUE FRANÇAISE}

Composantes harmoniques. Chez tous les sujets normaux on a calculé l'énergie des composantes harmoniques des réponses pour toutes les régions observées et pour tous les paramètres du stimulus. Il se trouve que chez tous les sujets normaux, les réponses sont composées principalement des premières et deuxièmes composantes harmoniques. La troisième composante ne fournit presque pas de contribution à la réponse. Des résultats similaires ont été obtenus pour toutes les régions analysées et pour tous les paramètres du stimulus appliqués de sorte que dans ce travail il ne sera pas question de la troisième composante harmonique. Le tableau I donne un résumé de ces calculs pour une fréquence de stimulation de $10 \mathrm{~Hz}$ et une profondeur de modulation de $30 \mathrm{p} .100$ pour la région occipitale droite et gauche chez les 5 sujets normaux.

Tableau I. - Région occipitale droite (D.) et gauche (G.) du scalp. Potentiels évoqués par L.M.S. Énergie (in $\%$ ) de la première $\left(A_{1}\right)$, deuxième $\left(A_{2}\right)$ et troisième $\left(A_{3}\right)$ composante harmonique chez des sujets normaux. Yeux ouverts.

Fréquence de L.M.S. : $10 \mathrm{~Hz}$. Profondeur de modulation 30 p. 100.

\begin{tabular}{lccccccc} 
Sujets Normaux & \multicolumn{2}{c}{$\mathrm{A}_{1}$} & \multicolumn{3}{c}{$\mathrm{A}_{2}$} & \multicolumn{3}{c}{$\mathrm{A}_{3}$} \\
& $\mathrm{D}$ & $\mathrm{G}$ & $\mathrm{D}$ & $\mathrm{G}$ & $\mathrm{D}$ & $\mathrm{G}$ \\
$18 / 70$ & 93.8 & 95.6 & 5.4 & 3.8 & 0.2 & 0.5 \\
$20 / 70$ & 93.2 & 90.4 & 6.5 & 9.3 & 0.1 & 0.2 \\
$22 / 70$ & 86.2 & 80.5 & 11.8 & 17.2 & 1.3 & 1.5 \\
$28 / 70$ & 79.0 & 78.5 & 18.7 & 19.6 & 0.6 & 0.5 \\
$29 / 70$ & 41.0 & 35.8 & 54.0 & 60.9 & 2.6 & 2.0
\end{tabular}

Influence de la profondeur de la modulation (P.M.). L'étude de l'influence des changements de la P.M. sur l'amplitude de la réponse globale «over-all» et des composantes harmoniques a montré qu'en général avec une petite P.M. des amplitudes plus petites sont obtenues qu'avec une grande P.M.

Dans certains cas, l'amplitude de la réponse globale « over-all » et de ses composantes harmoniques paraît diminuer avec une augmentation de la P.M. (« diminution paradoxale »). Ce phénomène apparaît tantôt bilatéralement tantôt unilatéralement. Dans ce cas il détermine une asymétrie. En outre, les amplitudes de la $1^{r^{\mathrm{e}}}$ et de la $2^{\mathrm{e}}$ composante harmonique ne sont pas toujours influencées de la même façon que celles de la réponse globale « over-all ». La première composante harmonique montre souvent la même image que la réponse globale «over-all», mais la $2^{\text {e }}$ composante harmonique peut se comporter de façon différente.

Un exemple illustrant ces observations, obtenu pour les régions occipitales droite et gauche, chez un des sujets normaux avec une fréquence de stimulation de $16 \mathrm{~Hz}$ et des P.M. de 10,30 et 50 p. 100 est montré dans la figure 3. Dans la colonne de gauche on voit qu'avec l'augmentation de la P.M. de 10 p. 100 à 30 p. 100 l'amplitude globale « over-all » $\left(A_{0}\right)$ augmente du côté droit et diminue du côté gauche ; par conséquent on constate une asymétrie de la réponse globale " over-all ».

L'amplitude de la $1^{\text {re }}$ composante harmonique $\left(A_{1}\right)$ au contraire diminue des deux côtés, tandis que l'amplitude de la $2^{\circ}$ composante harmonique $\left(A_{2}\right)$ augmente. La répétition de la même expérience (colonne 2 de la figure 3 ) chez le même sujet dans des situations aussi semblables que possible, montre que les amplitudes des réponses varient beaucoup.

Pour la $3^{\mathrm{e}}$ expérience, les amplitudes $\mathrm{A}_{\mathrm{o}}$ et $\mathrm{A}_{1}$ montrent une diminution à gauche et une aug- 

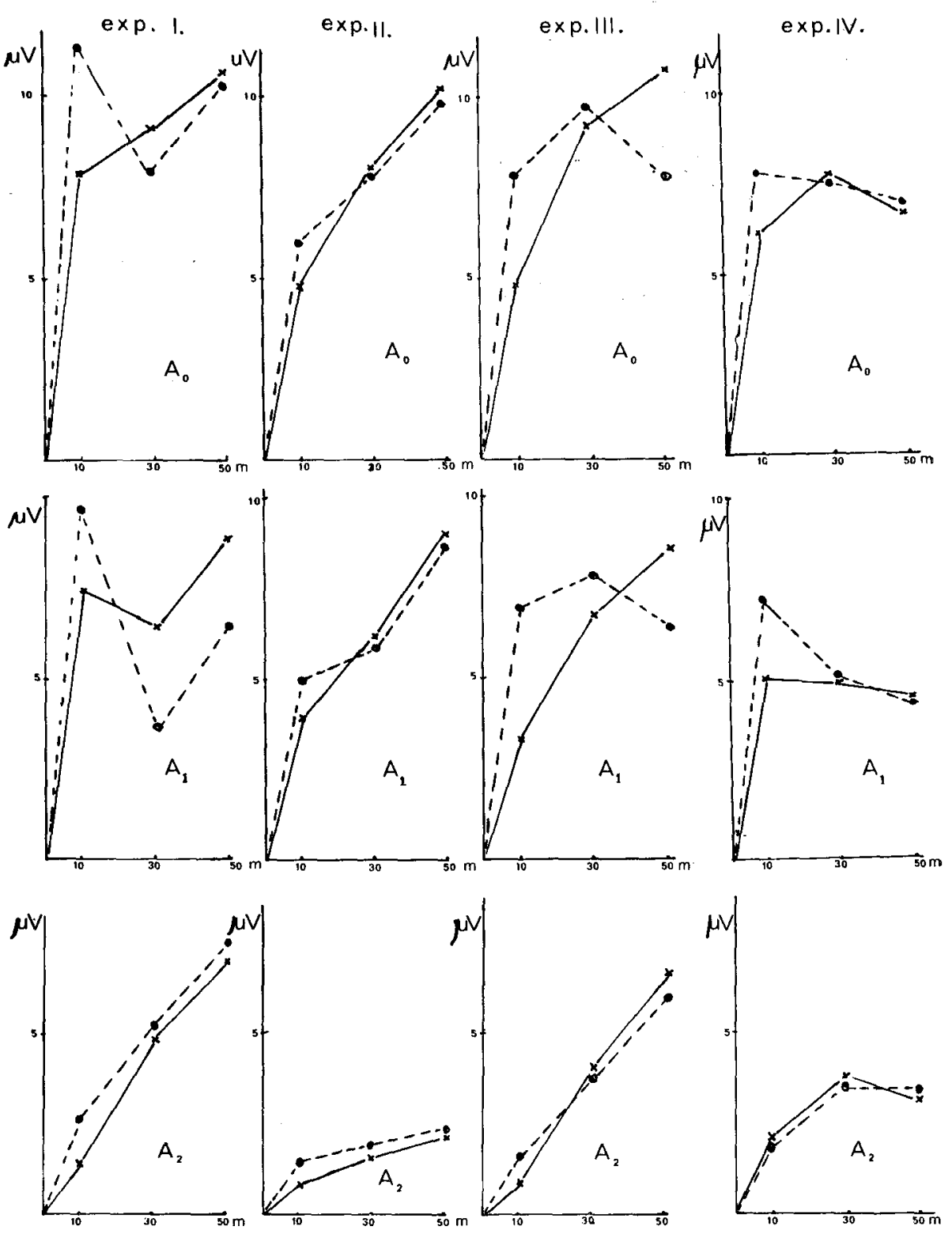

FIG. 3. - Région occipitale droite et gauche du scalp. Influence de la profondeur de modulation (M in \%) aux amplitudes (in $\mu V$ ) de la réponse totale $\left(A_{0}\right)$, de la première $\left(A_{1}\right)$ et deuxième $\left(A_{2}\right)$ composante harmonique chez un sujet normal.

$$
\begin{aligned}
& \mathrm{X}-ニ-ニ-ニ ニ-X \\
& \mathrm{O}-\mathrm{X} \text { droite }
\end{aligned}
$$

Résultats obtenus au cours de 4 expériences (I, II, III, IV) identiques. 
mentation à droite avec un changement de la P.M. de 30 p. 100 à 50 p. 100. L'amplitude $A_{2}$ augmente des deux côtés. La $4^{\mathrm{e}}$ expérience montre des résultats encore différents pour les amplitudes des réponses $A_{0}, A_{1}$ et $A_{2}$. Sur la figure il apparaît également qu'aussi bien du côté droit que du côté gauche la réponse globale « over-all " et les composantes harmoniques montrent des amplitudes très variables d'une expérience à l'autre. Chez tous les sujets normaux le comportement des réponses globales «over-all» et de leurs composantes harmoniques sous l'influence du changement de la P.M. est similaire pour les trois fréquences de stimulation utilisées et pour les trois régions symétriques du scalp explorées.

TABLEAU II. - Coefficients de variation calculés pour les composantes harmoniques chez des sujets normaux et des patients pour les régions occipitales droites et gauches. Fréquence de stimulation : $10 \mathrm{~Hz}$.
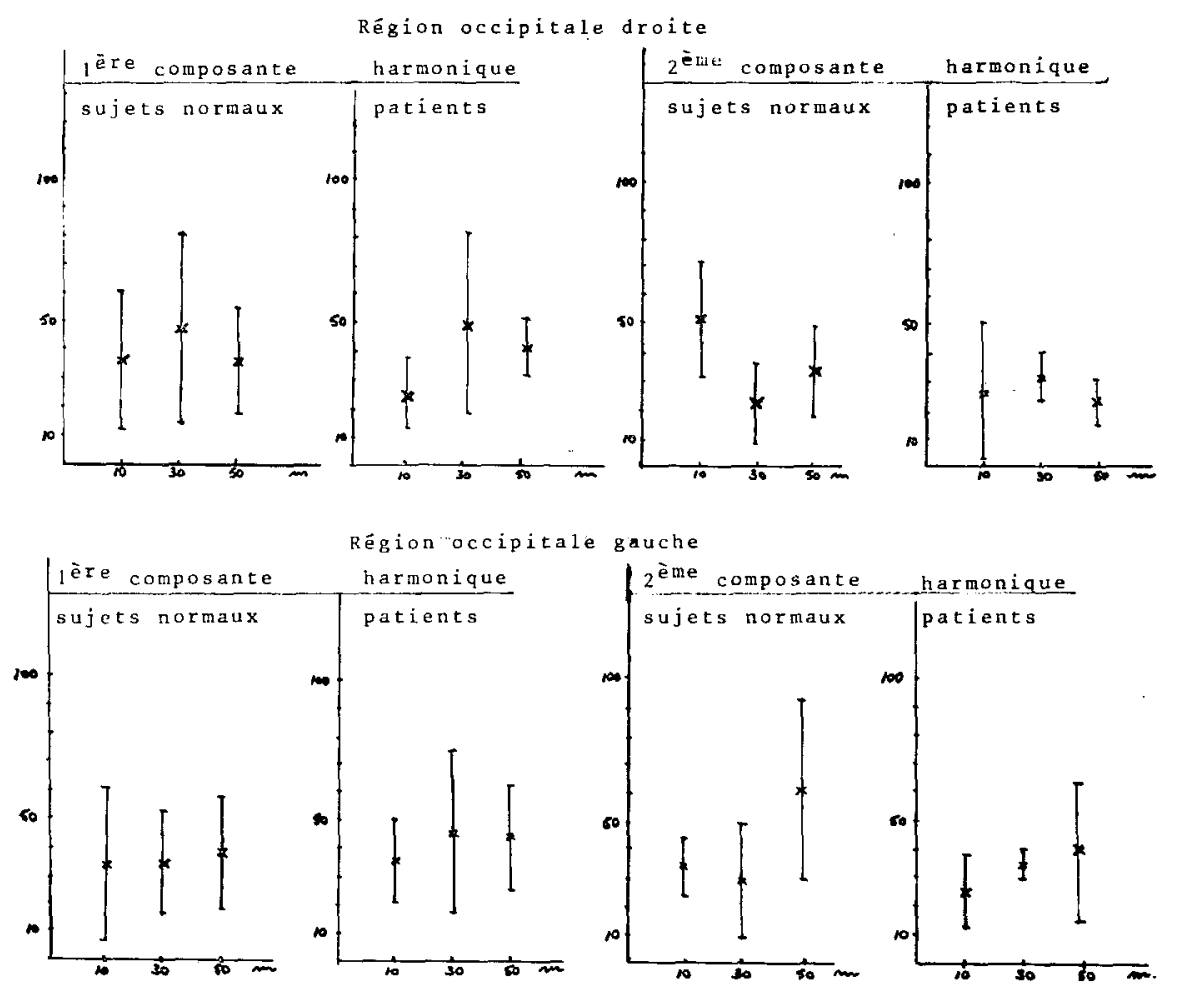

Variabilité des amplitudes. Chez tous nos sujets normaux la moyenne et l'écart type ont été calculés pour les amplitudes des réponses globales «over-all » et des composantes harmoniques, enregistrées sur les trois régions du scalp symétriques pendant 4 expériences identiques et ceci pour tous les paramètres utilisés. Ces calculs montrent que les variations des amplitudes sont très grandes. Une-grande variabilité intra-individuelle des réponses globales « over-all » et des composantes harmoniques a été trouvée pour chaque sujet normal examiné.

Pour les trois régions symétriques du scalp, chez tous les sujets normaux le coefficient de variation, c'est-à-dire l'écart-type calculé en pourcentages de la déviation moyenne, a été calculé pour les amplitudes de la $1^{\mathrm{re}}$ et de la $2^{\mathrm{e}}$ composante harmonique ; 
Le coefficient de variation moyen et son écart type, catculé pour le groupe total des sujets normaux, indique l'existence d'une grande variabilité inter-individuelle.

Le tableau II donne, entre autres, un résumé des coefficients de variation moyens et de leurs écarts-types pour tous les sujets normaux pour une fréquence de stimulation de $10 \mathrm{~Hz}$ et pour des P.M. de 10, 30 et 50 p. 100 dans le domaine occipital droit et gauche.

\section{Patients.}

Dans le groupe des patients on a trouvé des résultats qui sont les mêmes que ceux du groupe des sujets normaux. Dans ce groupe on retrouve également des différences de forme entre les réponses dans le domaine occipital et pariétal d'une part et dans le domaine temporal de l'autre.

Les réponses sont surtout composées des $1^{\mathrm{re}}$ et $2^{\mathrm{a}}$ composantes harmoniques. Ces résultats ont été retrouvés chez tous les patients et dans tous les domaines examinés.

Sous l'influence d'une augmentation de la profondeur de modulation, on constate en général une augmentation des amplitudes des réponses. Cependant le phénomène de diminution paradoxale, soit unilatéral, soit bilatéral, a été observé chez tous les patients pendant une ou plusieurs expériences.

L'influence des changements dans la profondeur de modulation est différente pour les composantes harmoniques des réponses et apparaît très variable comme dans le groupe des normaux.

Dans le groupe des patients on observe également une grande variabilité d'amplitude intra-individuelle et inter-individuelle.

Dans le tableau II sont résumés les coefficients de variations moyens et leurs écarts-types calculés pour le groupe des patients dans le domaine occipital, à droite et à gauche, avec une fréquence de stimulation de $10 \mathrm{~Hz}$ et une profondeur de modulation de 10,30 et $50 \mathrm{p} .100$.

Afin de déterminer s'il existe des différences significatives dans la variabilité des amplitudes, pour des domaines identiques, entre le groupe des sujets normaux, et celui des patients, on a cherché pour tous les paramètres de stimulus si les coefficients de variance différaient significativement (test $\mathrm{F}$ d'égalité des variances).

Pour 7 degrés de liberté et un niveau de signification de 5 p. 100 la valeur critique pour $F$ est : $5.59=\mathrm{Fc}$.

Dans le domaine occipital de l'hémisphère droit on a trouvé pour la première composante harmonique les valeurs $F$ suivantes :

$$
\text { Pour } \begin{gathered}
10 \mathrm{~Hz} 10 \%: 0.814 \\
10 \mathrm{~Hz} 30 \%: 0.001 \\
10 \mathrm{~Hz} 50 \%: 0.134
\end{gathered}
$$

et pour la $2^{\mathrm{e}}$ composante harmonique :

$10 \mathrm{~Hz} 10 \%: 3.450$

$10 \mathrm{~Hz} 30 \%: 1.560$

$10 \mathrm{~Hz} 50 \%: 1.570$

Dans le domaine occipital de l'hémisphère gauche les valeurs de $\mathrm{F}$ sont : pour la première composante harmonique :

$10 \mathrm{~Hz} 10 \%: 0.005$

$10 \mathrm{~Hz} 30 \%: 0.513$

$10 \mathrm{~Hz} 50 \%: 0.170$

et pour la deuxième composante harmonique :

$10 \mathrm{~Hz} 10 \%: 0.954$

$10 \mathrm{~Hz} 30 \%: 0.392$

$10 \mathrm{~Hz} 50 \%: 0.006$ 
Les valeurs trouvées sont toujours plus petites que Fc (5.59) ce qui veut dire qu'aucune différence significative n'a été trouvée en ce qui concerne les coefficients de variations des deux groupes dans les domaines occipitaux.

\section{Discussion.}

Cette étude montre qu'au moyen de la stimulation par L.M.S. avec une fréquence entre 9 et 16 $\mathrm{Hz}$ on trouve des réponses non seulement dans les régions occipitales mais aussi dans les régions temporales et pariétales. Ces réponses paraissent être composées surtout de la première et de la deuxième composante harmonique.

Aussi bien chez les sujets normaux que dans le groupe des patients le phénomène de diminution paradoxale a été observé tantôt bilatéral, tantôt unilatéral. Dans ce dernier cas les amplitudes des réponses diminuent de façon unilatérale avec une augmentation de la profondeur de modulation. Ceci a été constaté pour les amplitudes des réponses globales comme pour les amplitudes des composantes harmoniques.

Les expériences montrent que les asymétries ainsi observées, lesquelles ont été appelées par KAMPHUISEN « asymétries inconsistantes », sont très variables. En outre dans le groupe des sujets normaux comme dans le groupe des patients il existe une grande variabilité intra-individuelle des réponses à la L.M.S. et ceci dans tous les domaines explorés et pour tous les paramètres du stimulus.

Étant donné ces résultats et étant donné l'existence d'une corrélation entre la présence d'asymétries inconsistantes et la présence d'anomalies E.E.G. irritatives semblables à celles décrites par KAMPHUISEN, on pouvait s'attendre à ce que dans les cas des anomalies E.E.G. irritatives une plus grande variabilité des amplitudes soit également observée. Cependant aucune différence significative n'a été trouvée entre les coefficients de variation concernant les domaines homologues des deux groupes normaux et malades.

En outre la comparaison des coefficients de variation n'a pas non plus montré de différence significative entre les domaines symétriques et ceci pour les deux groupes examinés.

A partir de ces résultats, nous avons conclu que la variabilité intra-individuelle peut être la cause des asymétries inconsistantes, mais que la variance seule n'est pas une mesure utile dans le diagnostic clinique pour la distinction entre affections irritatives et affections non irritatives.

Il paraît important d'essayer de diminuer la variabilité des amplitudes en modifiant les conditions d'examens des sujets. C'est dans cette perspective que nous avons actuellement entrepris de nouvelles expériences.

\section{BIBLIOGRAPHIE}

Kamphuisen (H. A. C.). Average E.E.G. response to sinusoidally modulated light in normal subjects and patients. Thesis University of Utrecht, 1969.

Lopes DA Sieva (F. H.). Dynamic characteristics of visual evoked potentials. A quantitative study. Thesis University of Utrecht, 1970, $126 \mathrm{p}$.

Lopes Da Silva (F. H.), Tweel (L. H.), Van der, Kamphuisen (H. A. C.), Mol (J. F.) and Storm van LeEUWEN (W.). Symposium on the use of sinusoidally modulated light for the study of visual mechanism in normal subjects, patients and animals (dog). Electroenceph. Clin. Neurophysiol., 1967, 23, 596-597.

REGAN (D. A.). A high frequency mechanism which underlies visual evoked potentials. Electroenceph. Clin. Neurophysiol., 1968, 25, 231-237.

Regan (D. A.) and Heron (J. R.). Clinical investigation of lesions of the visual pathway: a new objective technique. J. Neurol. Neurosurg. Psychiat., 1969, 32, 479-483.

SPEKREYSE (H.). Analysis of E.E.G. responses in man, evoked by sine wave modulated light. Thesis University of Amsterdam 1966, 163 p.

Tweel (L. H., van der), Verduyn Lunel (H. F. E.). Human visual responses to sinusoidally modulated light. Electroenceph. Clin. Neurophysiol., 1965, 18, 587-598. 